\title{
Setups for alignment and on-ground calibration and characterization of the EnMAP hyperspectral imager
}

Leonhard Polz, Anna Serdyuchenko, M. Lettner, M. Mücke, S. Fischer

Leonhard Polz, Anna Serdyuchenko, M. Lettner, M. Mücke, S. Fischer, "Setups for alignment and on-ground calibration and characterization of the EnMAP hyperspectral imager," Proc. SPIE 11852, International Conference on Space Optics - ICSO 2020, 118526B (11 June 2021); doi:

$10.1117 / 12.2600240$

SPIE Event: International Conference on Space Optics - ICSO 2021, 2021, Online Only 


\section{International Conference on Space Optics-ICSO 2020}

Virtual Conference

30 March-2 April 2021

Edited by Bruno Cugny, Zoran Sodnik, and Nikos Karafolas
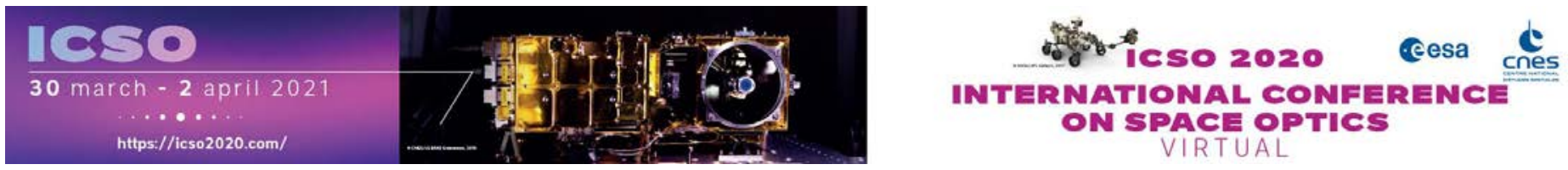

Setups for alignment and on-ground calibration and
characterization of the EnMAP hyperspectral imager

\section{Cesa isoporeseatings denes}




\title{
Setups for alignment and on-ground calibration and characterization of the EnMAP Hyperspectral Imager
}

\author{
Leonhard Polz*a, Anna Serdyuchenko ${ }^{\mathrm{a}}$, M. Lettner ${ }^{\mathrm{a}}$, M. Mücke ${ }^{\mathrm{a}}, \mathrm{S}$. Fischer ${ }^{\mathrm{b}}$ \\ ${ }^{a} O H B$ System AG, Manfred-Fuchs-Str. 1, 82234 Weßling, Germany; \\ bDLR Space Administration, Königswinter Str. 522-524, 53227 Bonn, Germany
}

\begin{abstract}
The Environmental Mapping and Analysis Program HyperSpectral Imager (EnMAP HSI) will allow to acquire images of the Earth surface in a push-broom configuration. Spectral coverage includes 230 wavelength bands between $420 \mathrm{~nm}$ and $2450 \mathrm{~nm}$, which are simultaneously recorded with a ground resolution of $30 \mathrm{~m}$ x $30 \mathrm{~m}$.

High requirements on the optical alignment of the Visual Near InfraRed (VNIR) and Short Wavelength InfraRed (SWIR) spectrometers and cameras have been fulfilled (one pixel lateral alignment and below $20 \mu \mathrm{m}$ for defocus). In addition, a calibration and characterization campaign of the EnMAP HSI was performed, with the aim to verify the instrument requirements and to quantify its performance. The campaign addressed the following five responsivity domains: radiometric, polarization, spectral responsivity, spatial responsivity and stray light. A spectral calibration accuracy of $0.5 \mathrm{~nm}$ for VNIR and $1 \mathrm{~nm}$ for SWIR channel, and lateral calibration of the full field-of-view with sub-arc second accuracy was achieved. For all measurements, traceability to national standards is guaranteed by comparative measurements with calibrated devices.

The paper presents the setups of the alignment, calibration and characterization measurements of the EnMAP HSI. Instrument requirements on calibration accuracy are reported. The traceability to national standards will be described and the validation of measurement accuracy.
\end{abstract}

Keywords: EnMAP, hyperspectral, OGSE, alignment, calibration, characterization, optical payload

\section{INTRODUCTION}

The Environmental Mapping and Analysis Program (EnMAP) is a German space borne science mission that aims at characterizing the Earth's environment on a global scale. The applications range from climate change impacts, the monitoring of large ecological systems, land cover changes, biodiversity processes and natural resources, to geohazard and risk assessments [1]. The single payload of the satellite is the Hyper Spectral Imager (HSI). It is capable of measuring the solar radiance reflected from the Earth's surface as a continuous spectrum in the spectral range of $420 \mathrm{~nm}$ to $2450 \mathrm{~nm}$.

\subsection{The EnMAP Instrument}

The EnMAP HSI is a state-of-the-art satellite-based hyperspectral Instrument for Earth observation in push broom configuration. The full spectral range is measured with two separate spectrometers, VNIR and SWIR, which share the same telescope, but have different line of sight. In both spectrometers the EnMAP swath width of $30 \mathrm{~km}$ is sampled in spatial direction with $30 \mathrm{~m}$ ground sampling distance. The main performance parameters are summarized in Table 1.

*Leonhard.Polz@OHB.de; phone +49 8153 4002-635; fax +49 8153 4002-941; www.OHB.de 


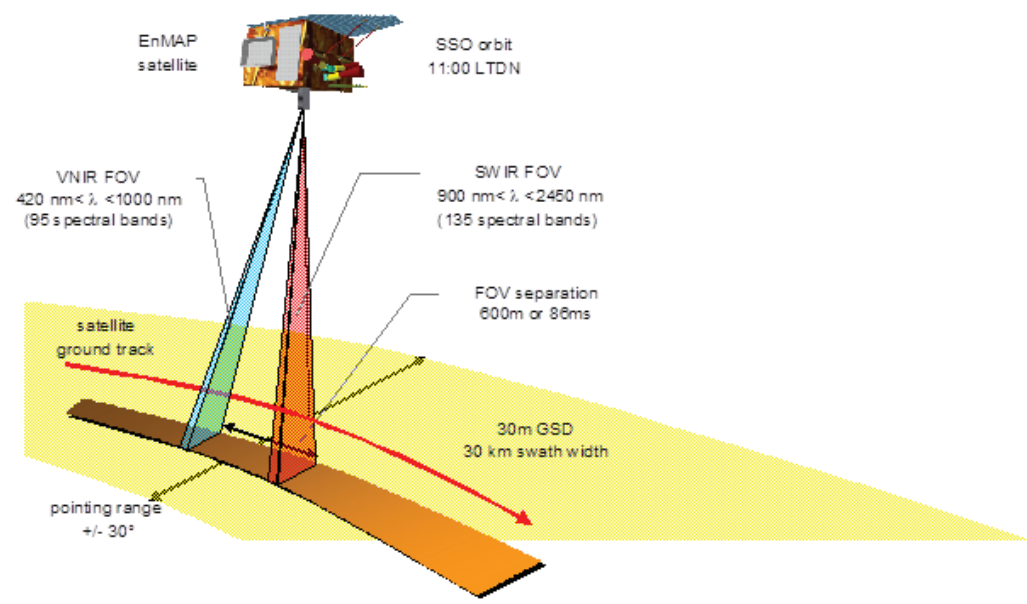

Figure 1. EnMAP Field Of View (FOV) and overpass concept.

Table 1. Key specifications for the EnMAP instrument.

\begin{tabular}{|ll|}
\hline \multicolumn{2}{|c|}{ EnMAP key specifications } \\
Parameter & value \\
\hline Instantaneous field of View (IFOV) & 9.5 arcsec \\
Instrument field of View (FoV) & $2.63^{\circ}$ (across track) \\
Spectral range & $420 \mathrm{~nm}-2450 \mathrm{~nm}$ \\
Spectral sampling distance (SSD) & VNIR $4.7 \mathrm{~nm}-8.3 \mathrm{~nm}$ \\
& SWIR $7.4 \mathrm{~nm}-12.1 \mathrm{~nm}$ \\
Bandwidth & 1 to $1.25 * \mathrm{SSD}$ \\
Spectral smile & $<20 \%$ of SSD \\
Spectral calibration accuracy & VNIR $0.5 \mathrm{~nm}$ \\
& SWIR $1 \mathrm{~nm}$ \\
Radiometric accuracy & $<5 \%$ \\
Radiometric stability & $<2.5 \%$ \\
Smile / Keystone & $<0.2$ pixel \\
Polarization sensitivity & $<5 \%$ \\
\hline
\end{tabular}

Figure 2 shows a cross-section view of the HSI. The instrument features a nearly diffraction-limited three-mirror anastigmat telescope assembly (TA) with an across-track Field Of View (FOV) of $2.63^{\circ}$. The TA has a $180 \mathrm{~mm}$ entrance aperture and focuses the light from the earth's surface onto a field splitter slit assembly (FSSA). The FSSA includes two separate micro slits for in-field separation of the light and a micro-mirror to redirect the SWIR field into the SWIR spectrometer [2]. Both, VNIR and SWIR spectrometer optics employ curved prisms in dual-pass configuration as dispersive elements. Light from the spectrometer is finally focused on VNIR and SWIR focal planes arrays that acquire images at a frame rate of $230 \mathrm{~Hz}$ with 14-bit resolution.

All optical elements are mounted to a monolithic 3-dimensional Aluminum structure. The Aluminum structure together with the Spectrometers and FSSA forms the Instrument Spectrometer Unit (ISU). To avoid a loss of optical performance due to the thermal expansion of the Aluminum structure, the whole HSI is temperature controlled by a Thermal Control SubSystem (TCSS) to $21^{\circ} \mathrm{C}$.

For fulfilling the stringent requirements on overall pointing stability, the star tracker sensor assembly is directly attached to the optical unit. In addition, an on-board calibration system will be used for recurring in-flight radiometric and spectral calibration. It consists of a solar calibration assembly containing a diffuser which reflects sunlight into the Telescope entrance aperture as well as a calibration light source (not shown in Figure 2) that can be fed into the beam path in front of the Spectrometer entrance slits [1]. The calibration light source is used to monitor the calibration validity at small time 
intervals, whereas the solar calibration assembly will be used less frequently, but as the primary radiometric calibration device during space operation.

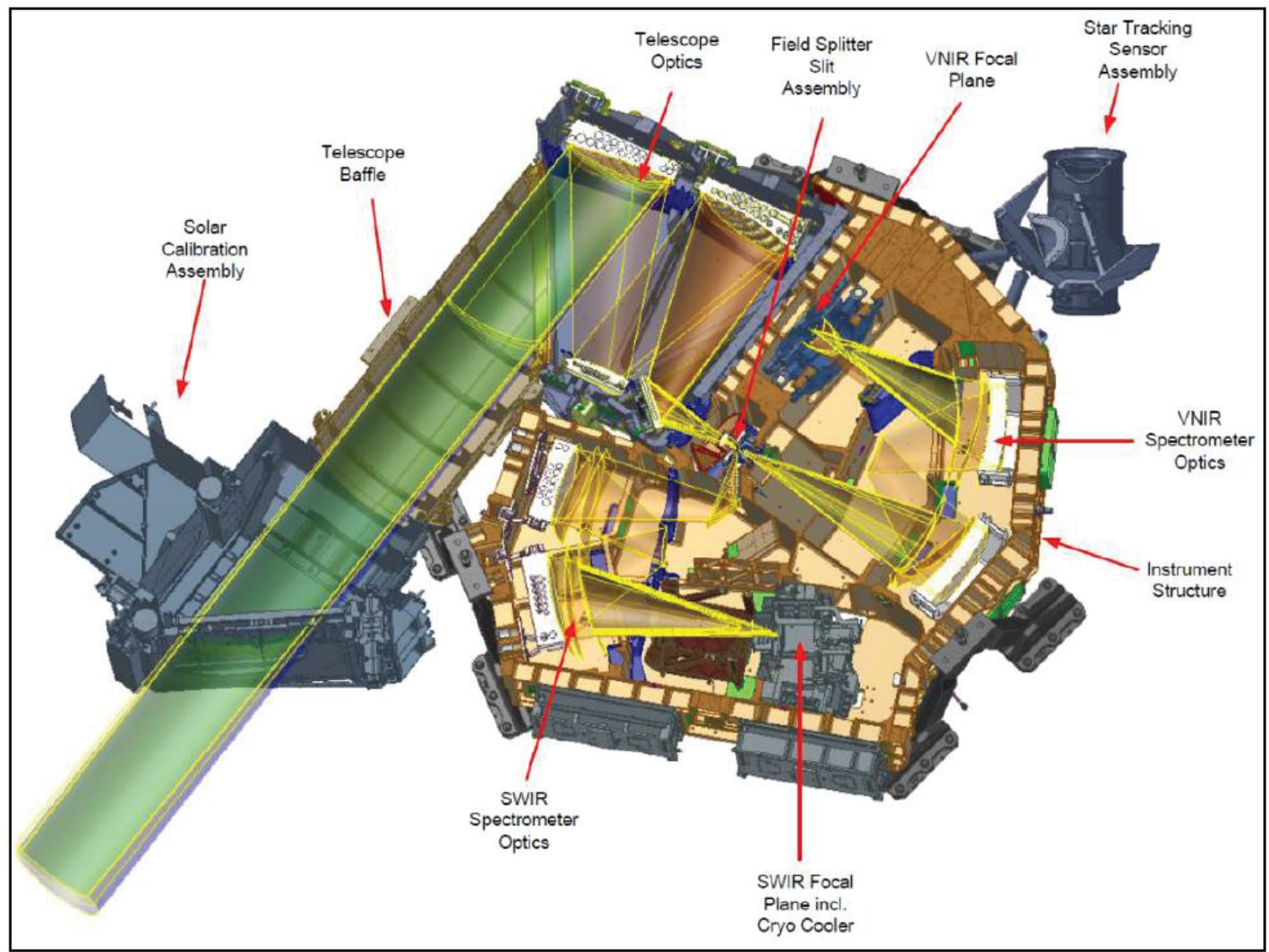

Figure 2. Cross-section of the EnMAP HSI main optical units

\subsection{General setup aspects}

Aligning and calibrating a state-of-the-art hyperspectral instrument as the EnMAP HSI requires to establish measurement setups that outperform the test object in all relevant performance aspects to achieve the required measurement accuracies. At the same time technical as well as economic considerations yield to develop measurement equipment that can support multiple use cases throughout the Alignment integration and Test (AIT) Process of EnMAP and future projects. In this spirit, the following main aspects were considered.

\section{$\underline{\text { Cleanroom and monitoring }}$}

The EnMAP HSI was integrated and aligned in an ISO5 class cleanroom with a size of $150 \mathrm{~m}^{2}$. The room temperature can be set to different temperatures, depending on the needs of the project, and was stabilized to a temperature of $21^{\circ} \mathrm{C} \pm 0.5^{\circ} \mathrm{C}$ for the AIT process of the EnMAP HSI. HSI and Optical Ground Support Equipment (OGSE) were extensively monitored with temperature sensors, to validate that the instrument as well as the OGSE stayed in this temperature range during all alignment and calibration measurements. Additionally, also the absolute barometric pressure was measured close to the instrument, to be able to correct the data for air-to-vacuum shifts. To monitor the cleanliness status during all activities, short term and long term whiteness samples were used and analyzed on a regular basis. Especially for alignment and geometrical calibration measurements a good stability of the setup was necessary. To this end, the room was equipped with a massive $30 \mathrm{~m}^{2}$ granite block, which easily accommodate all EnMAP OGSE setups. 


\section{$\underline{\text { Modular OGSE approach }}$}

For higher flexibility in the AIT process, a modular OGSE approach was chosen, with separated light sources and illumination optics [3]. As a connection between both, standardized fibre optic interfaces have been used. This was done to maximize synergies of recurring tasks and requirements between spectral and geometrical characterisation as well as certain alignment activities. In this way, also heat transfer and cleanliness classes of the light sources did not impact. In addition, it allowed to adapt the AIT sequence to the availability of GSE and flight hardware and to optimize the schedule.

During calibration and characterization, as well as for the TA to Spectrometers alignment, Full aperture illumination of the Telescope is needed. It was thus a natural decision to look for a versatile OGSE solution that could already be employed during the alignment of the TA to the Spectrometers, but also later for geometrical, spectral and stray light measurements.

The reduction of the overall amount of OGSE items for alignment and test had a clear commercial advantage. In addition, the AIT team benefits from working with a multi-purpose setup. It allowed thorough understanding of the systematic behaviour and technical peculiarities of the involved devices, which is of importance, especially for calibration measurements, which require high setup stability, repeatability and accuracy.

\section{$\underline{\text { Automatization }}$}

Especially during the calibration and characterization campaign a huge amount of measurements were taken (several thousand data points). This was only possible, by a huge degree of automatization of the measurements. Therefore, all OGSE were equipped with remote command functions and network connections. The OGSE was remotely controlled using a central control authority, the GSE Operating System (GSEOS), which also controlled the HSI focal plane detectors. In this way, time synchronized batch script operation involving flight detectors and OGSE was possible. Synchronization lags were typically $<10 \mathrm{~ms}$ between the individual devices, which was sufficient for the envisaged use cases.

\section{ALIGNMENT}

The components of the HSI have been aligned and characterized consequently, starting with spectrometers, which have been aligned before the integration of the telescope assembly. The cameras have been aligned after the integration and alignment of the telescope assembly with high requirements on the optical alignment of both cameras have been fulfilled (1 pixel lateral alignment and below $20 \mu \mathrm{m}$ for defocus).

\subsection{Spectrometer alignment}

The objective of the spectrometer alignment was the optimization of the Wave Front Error (WFE) after interface generation [4] and the placement of the optical elements (OE). The alignment process accounted for the discrepancy between desired and actual positions of the OEs due to the manufacture and placement tolerances. The overall flow of the process:

- $\quad$ Optical characterization of the WFE;

- Computation of the relative correction of the adjustable OE;

- Correction of OE position;

- Control measurement of the correction;

- Iteration of the process until required WFE is achieved.

The alignment was performed for the VNIR and SWIR spectrometers separately. The WFE was measured at several field points and several discrete wavelengths in double-pass configuration with the setup depicted in Figure 3. A discrete wavelength light source equipped with 5 low coherence laser sources was coupled via fiber to the Spectrometer Spot Illumination and Detection (SpecSID) GSE. The SpecSID allowed to illuminate the entrance slit of the spectrometer at different positions with a well-defined spot illumination. From the spectrometer focal plane, the light was reflected back using a SpecSID Wavefront Reference (SIDWaRe) GSE to realize a double-pass WFE measurement. The double-pass WFE was measured using a Wavefront sensor inside SpecSID. 


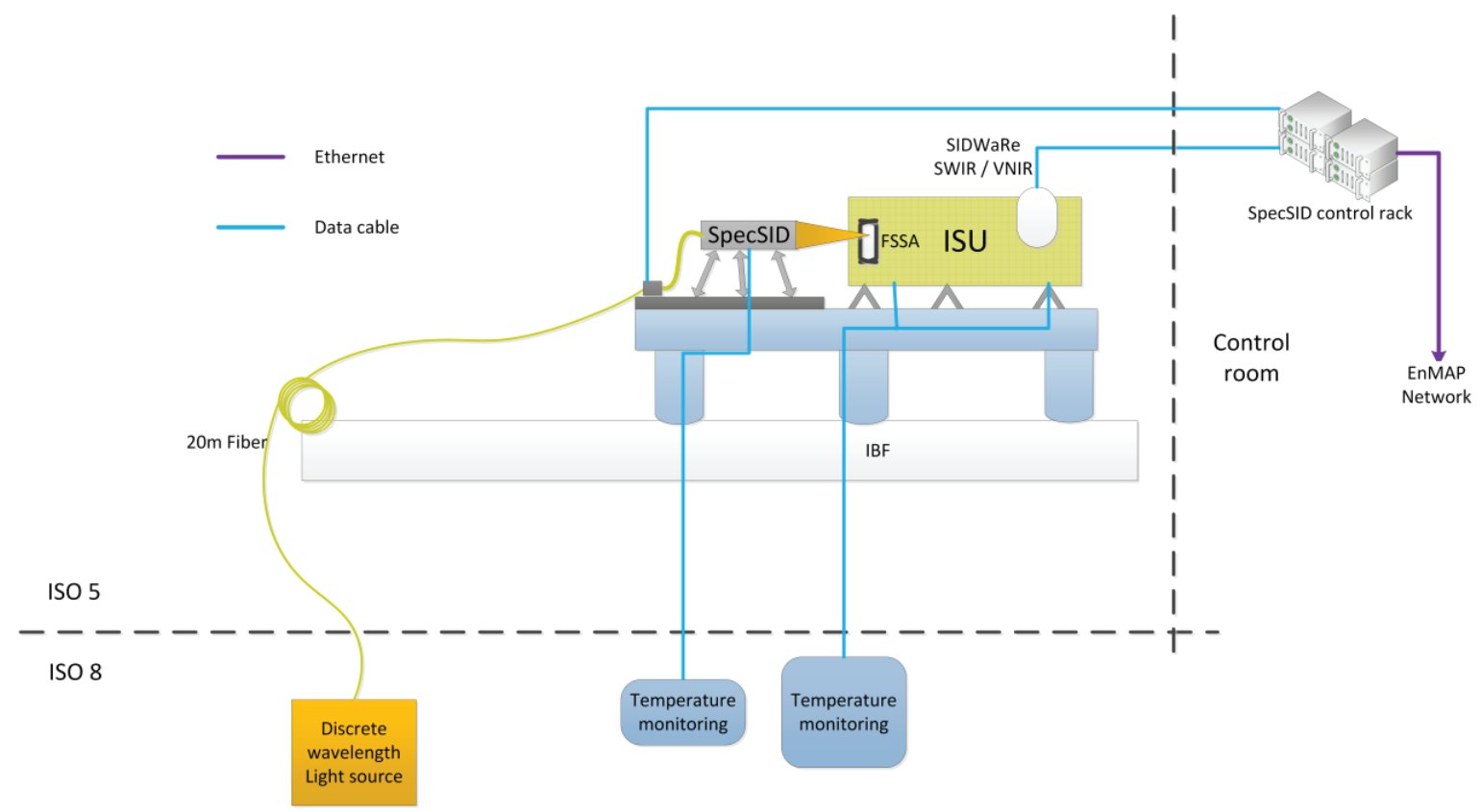

Figure 3. Setup for Spectrometer alignment

\subsection{Telescope assembly (TA) to Instrument Spectrometer Unit (ISU) alignment}

At first, the EnMAP telescope assembly was integrated, aligned and characterized by OHB on sub-assembly level with residual (single pass) wave front error of $28 \mathrm{~nm} \mathrm{rms} \mathrm{[5].} \mathrm{The} \mathrm{complete} \mathrm{assembly} \mathrm{was} \mathrm{later} \mathrm{aligned} \mathrm{to} \mathrm{the} \mathrm{ISU} \mathrm{forming} \mathrm{the}$ HSI.

Figure 4 shows the setup for this TA to ISU alignment. The TA entrance pupil was illuminated with a collimated light beam of $200 \mathrm{~mm}$ diameter coming from the Full Aperture Illuminator (FAI) [3]. The light was focused by the TA onto the FSSA and was reflected back by micro-mirrors on the FSSA [2]. There were six mirrors corresponding to extremal field points.

The FAI was developed, manufactured, integrated, aligned and tested upon OHB Specifications by Bertin Technologies. It was composed of a two-mirrors unobscured collimator, a scene generator and a source assembly. The source assembly included an internal light source and allowed to analyze the reflected light for collimation. Using a scanning system manufactured by Symetrie, the collimator was displaced angularly to address the six mirrors on the FSSA. The TA was mounted on a Telescope to ISU Integration GSE (TIIG), which moved the TA in front of the Spectrometer until the best focus position of the TA regarding all six micro mirrors was derived with an accuracy of ca. $1 \mu \mathrm{m}$ in focus direction. In this optimized position, the necessary shim thicknesses were determined. A displacement probe GSE helped to reference the TA position during shim integration. 


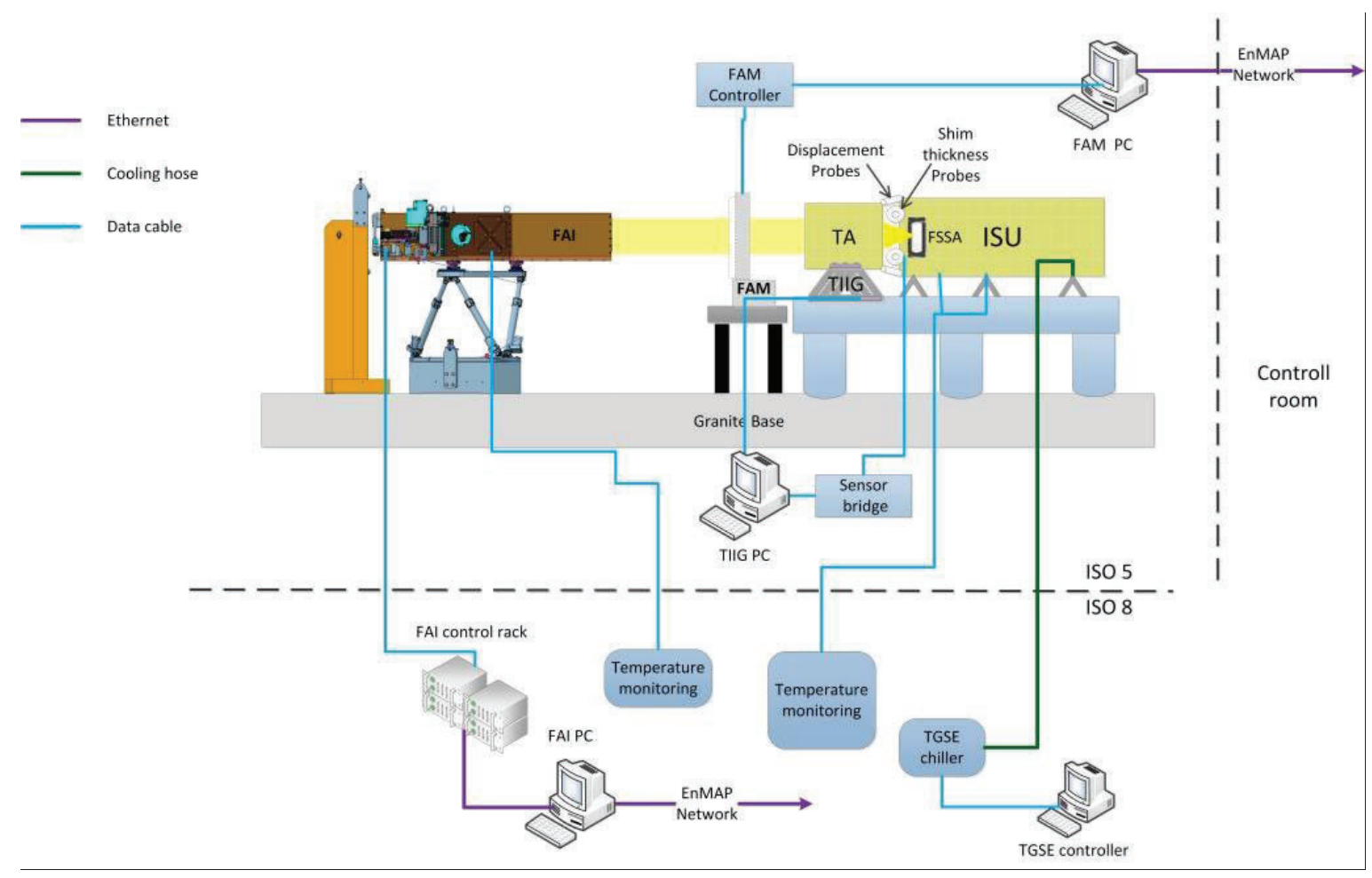

Figure 4. Setup for the Telescope to Instrument Spectrometer Unit alignment

\subsection{Camera alignment}

For the optical alignment of the cameras, a monochromatic spot illumination of the spectrometer slit was used, similar to the Spectrometer and the TA to ISU alignment. The light was detected directly by the flight models of the cameras, using the supporting Instrument Central Processing Unit (ICPU) and GSEOS.

For both channels, VNIR and SWIR, the same activities have been performed. First, an approximate lateral position of the camera has been found. After that focal position has been adjusted, optimizing the Modulation Transfer Function values (MTF) obtained from a series of measurements at different spectral and lateral fields. Finally, lateral position was updated to optimize usage of the detector active area and to minimize keystone.

The availability of both, SpecSID and FAI OGSE, made it possible to keep the whole alignment process flexible and allow for parallel work on the ISU and TA. According to schedule demands, the VNIR Camera was aligned and characterized using the SpecSID without the TA whereas the SWIR Camera alignment was done using the FAI after the TA final integration.

\section{CALIBRATION AND CHARACTERIZATION CAMPAIGN}

To verify the system performance on ground as well as to acquire relevant calibration data, an extensive on-ground Calibration and Characterization $(\mathrm{CnC})$ campaign was performed employing various OGSE setups.

The on-ground calibration is important for evaluation of those parameters, which cannot be measured in orbit. It is also essential to perform "end-to-end" measurements in order to test the whole instrument chain. 
The $\mathrm{CnC}$ measurements have been performed in three main blocks using a dedicated OGSE setup for each block: radiometric test, geometrical and spectral calibration and stray light test. The main measurement tasks of these three blocks are summarized in Table 2.

Table 2. Calibration and Characterization test blocks.

\begin{tabular}{|c|c|c|}
\hline $\begin{array}{l}\text { Radiometric and Polarization } \\
\text { setup } \\
\text { - } \quad \text { Radiometric response } \\
\text { - } \quad \text { Response linearity } \\
\text { - } \quad \text { Dark signal } \\
\text { - } \quad \text { Signal to Noise Ratio (SNR) } \\
\text { - } \quad \text { Polarisation sensitivity }\end{array}$ & $\begin{array}{l}\text { Geometrical and Spectral setup } \\
\text { - } \quad \text { Pixel center wavelength } \\
\text { - } \quad \text { Bandwidth } \\
\text { - } \quad \text { Smile } \\
\text { - } \quad \text { Spectral response function } \\
\text { - } \quad \text { Line of Sight (LoS) of individual } \\
\text { - } \quad \text { pixels } \\
\text { - } \quad \text { Modulation Transfer Function } \\
\text { - } \text { (MTF) } \\
\text { - } \quad \text { Line spread function (LSF) } \\
\text { - } \text { across and along track } \\
\text { aligntrument/star sensors }\end{array}$ & $\begin{array}{l}\text { Stray light setup } \\
\text { - } \quad \text { Spectral in band ghosts } \\
\text { - } \quad \text { Spatial in band ghosts } \\
\text { - } \quad \text { Diffuse in band stray light } \\
\text { - } \quad \text { Out of field stray light }\end{array}$ \\
\hline
\end{tabular}

\subsection{Radiometric and Polarization setup}

Although a radiometric characterization was already performed on detector level, it was repeated on instrument level during the $\mathrm{CnC}$ campaign, to achieve true end-to-end calibration data for the instrument.

The setup is depicted in Figure 5. The main components are:

- $\quad$ Light source: Fully Integrating Sphere (FIS), property of the Remote Sensing Technology Institute of the German Aerospace Center (DLR-IMF) [6];

- $\quad$ Calibrated Transfer Spectrometer (TS), which was connected to the FIS close to its aperture via 1m optical fibre;

- $\quad$ Rotatable Full Aperture Polarizer (FAP);

The FIS had a diameter of $1.6 \mathrm{~m}$ and delivered homogenous broadband illumination. It was capable of illumination at 14 different power levels, which was of importance especially for HSI linearity analysis and SNR measurements. The FIS aperture of $200 \mathrm{~mm} \times 300 \mathrm{~mm}$ was marginally bigger than the instrument FOV. For an exact absolute radiometric calibration of every pixel, the HSI FOV had to be covered without clipping. For this reason, the FIS aperture was aligned with respect to the HSI FOV with accuracy of $2 \mathrm{~mm}$. The radiance homogeneity within the FIS aperture was characterized in advance to be better than $0.3 \%$ peak to valley [7].

In Figure 6, the full radiometric calibration chain is depicted: The Transfer Spectrometer (TS) was used as a calibrated radiometric reference for all measurements. The TS itself was calibrated before and after the campaign using the RAdiometric STAndard (RASTA) at DLR-IMF, which is itself calibrated to national standards at the National Metrology Institute of Germany (PTB). With this calibration chain, a final radiometric measurement accuracy of $<1 \%$ standard deviation was achieved. 
To characterize the polarization sensitivity, a rotatable full aperture polarizer (FAP) with $280 \mathrm{~mm}$ clear aperture was placed and aligned between FIS and EnMAP HSI. The ultra-broadband polarizer guaranteed a degree of polarization of better than 1000 within the whole EnMAP range of $420 \mathrm{~nm}$ to $2450 \mathrm{~nm}$.

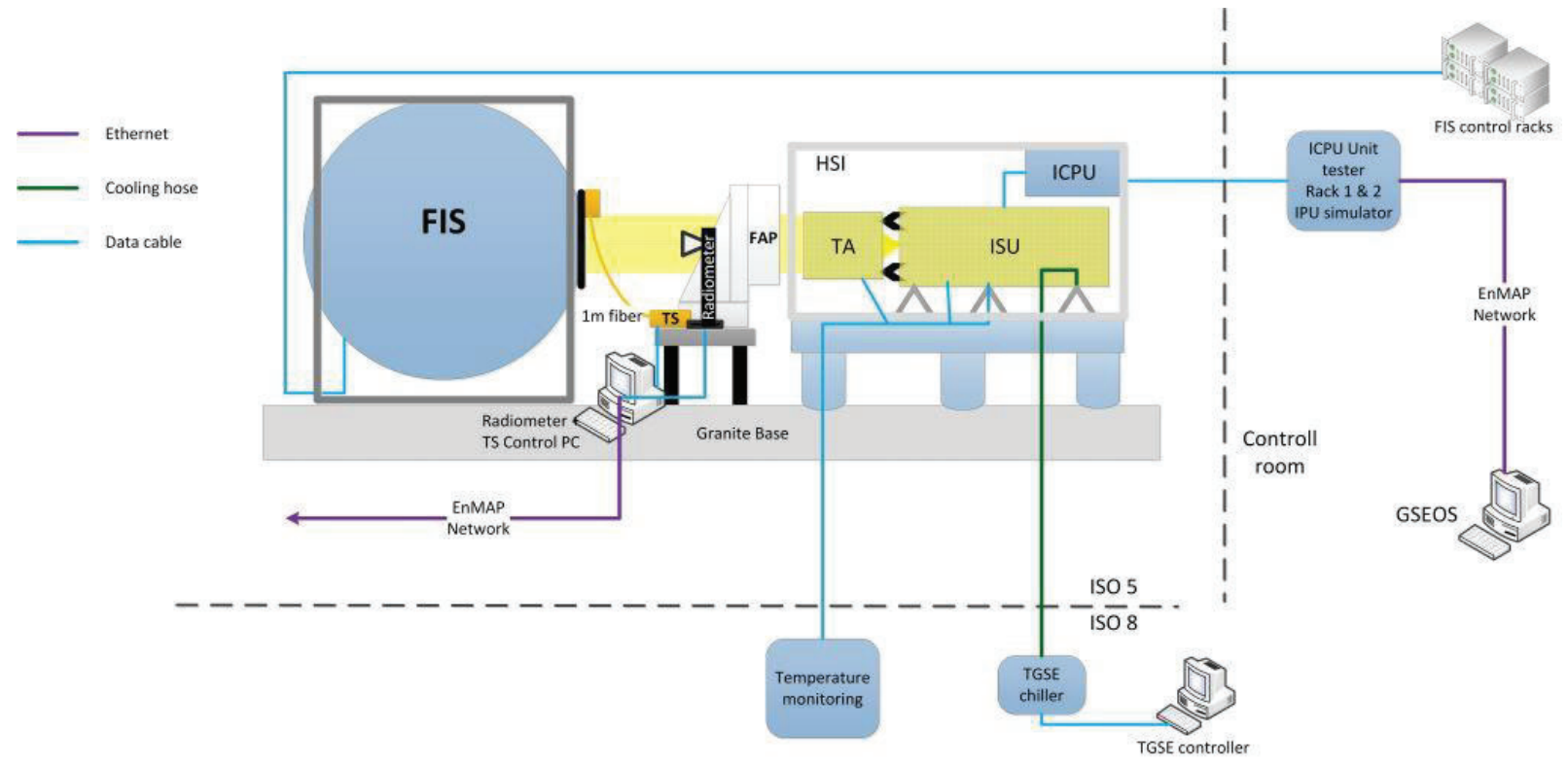

Figure 5. Setup for radiometric calibration, polarization sensitivity characterization and SNR measurements.

\section{Transfer}

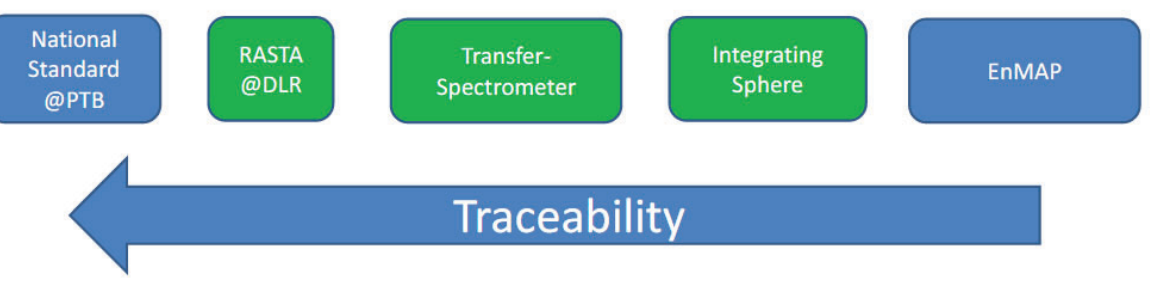

Figure 6. Tracability chain for the absolute radiometric calibration of EnMAP [7].
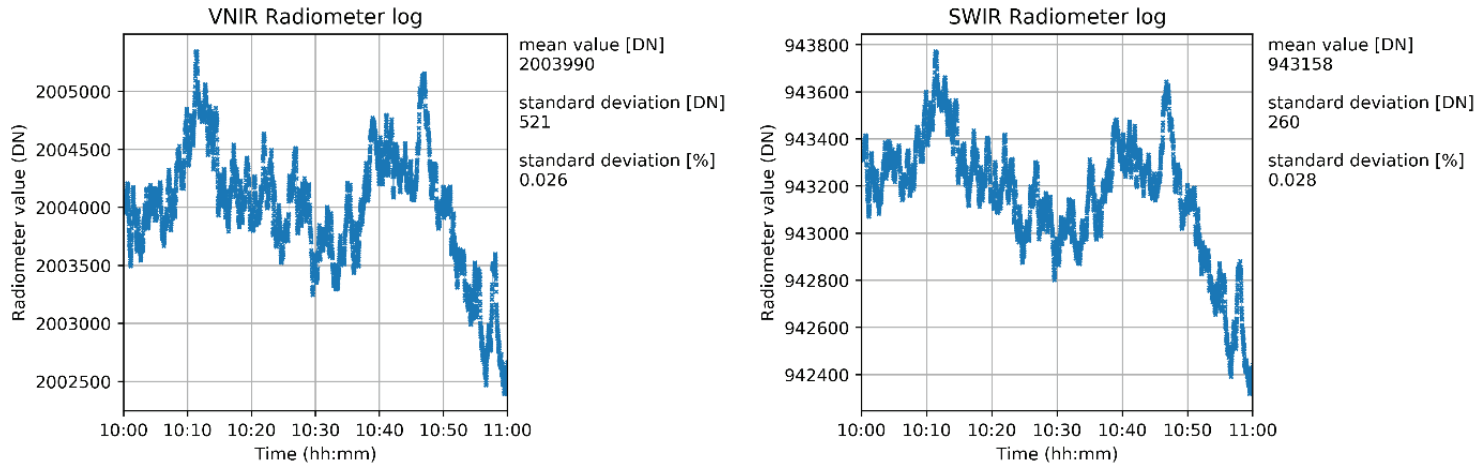

Figure 7. Radiance stability of FIS measured in the VNIR and SWIR range over 1 hour. 
The stability of FIS radiation, especially during the longer SNR and Polarization measurements, was monitored using two high sensitive Si and InGaAs radiometers, for the VNIR and SWIR range, respectively. Figure 7 shows the data of both radiometers during a time period of one hour at FIS level number 5 . The data shows excellent stability of $<0.03 \%$ standard deviation in both wavelength ranges. With the radiometers and the FAP, also the inherent degree of polarization of the FIS was validated to be smaller than $0.8 \%$.

\subsection{Geometrical and spectral setup}

Figure 8 shows the setup for the geometrical and spectral measurements. During the geometrical and spectral measurements, the HSI was illuminated using the FAI combined with the Wide Range Adjustable Light source (WiRAL), which was coupled to the FAI via optical fibres. To homogenize the HSI pupil illumination, a Fiber based Mixing System (FMS) was introduced in the optical fibre connection between WiRAL and FAI.

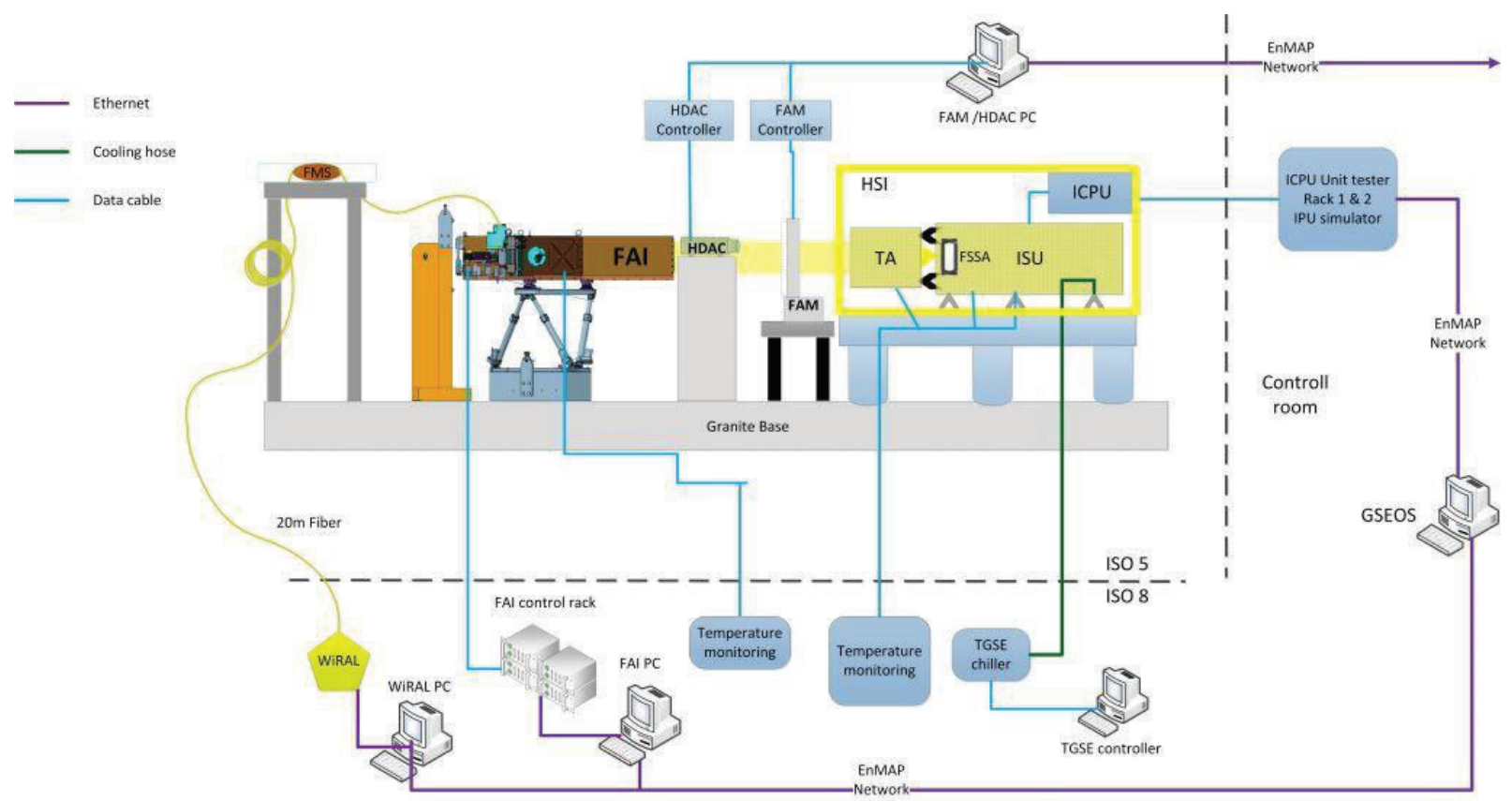

Figure 8. Setup for geometrical and spectral measurements

The core of the WiRAL is a commercially available Grating Monochromator (iHR320) by HORIBA Scientific, fed by several light sources, which was extensively characterized and optimized by OHB. It allowed adjustable monochromatic illumination in the complete EnMAP range from $420 \mathrm{~nm}$ to $2450 \mathrm{~nm}$ and even beyond, with a bandwidth of $0.5 \mathrm{~nm}$ to $1 \mathrm{~nm}$, which was smaller than $10 \%$ of the instrument spectral sampling distance.

WiRAL was calibrated traceable to national standards using a PTB-calibrated Echelle spectrometer provided by DLRIMF. It had a maximum wavelength uncertainty of $0.005 \mathrm{~nm}$ at $1000 \mathrm{~nm}$ and $0.0125 \mathrm{~nm}$ at $2500 \mathrm{~nm}$ [8], which was far below the $\mathrm{CnC}$ requirement of $0.1 \mathrm{~nm}$ in the range of $420-1000 \mathrm{~nm}$ and $0.2 \mathrm{~nm}$ in the range of $1000-2450 \mathrm{~nm}$. Additionally, a dedicated recurring calibration plan employing Gas-Cell line sources ensured the validity of the calibration throughout the complete EnMAP on-ground calibration campaign.

By scanning the WiRAL wavelength and synchronous image takes of the instrument detectors, the spectral response function of all spectral pixels of several across-track pixel rows were obtained. Since the FAI illuminated the HSI with collimated light, a sufficient intensity level on the detector was easily achieved, but only a few pixels in across-track direction could be illuminated at the same time. Hence, after each wavelength scan, the FAI had to be moved angularly with its scanning system to address the next row of pixels, resulting in a very long measurement series. However, the 
implemented automatization capabilities described in section 1.2 enabled scripted measurement sequences controlled by the GSEOS software which run independently overnight without operator control. The geometrical measurements, which cannot be automatized in such a high degree, were performed in parallel during the daytime. This enabled to keep the tight $\mathrm{CnC}$ campaign schedule without compromises on the desired amount of measurements and without the need of shift work.

For the geometrical measurements, knife edge structures situated in the scene generator of FAI were imaged onto the instrument detectors and stepped across the illuminated field in sub pixel steps. Detector images were synchronously taken using the instrument detectors, to determine MTF and instantaneous FOV and to correlate the pixel centre with the knife edge position [3].

To correlate the HSI LoS with the FAI knife-edge, a special method has been used (a detailed description of the procedure can be found in [3]). The method implied a Full Aperture Mirror (FAM) aligned in front of FAI and a High Dynamic AutoCollimator (HDAC) aligned on the HSI reference cube. The FAM and the HDAC linked the optical axis of the FAI collimator with the HSI optical axis. The HDAC is a customized electronic autocollimator developed by Möller-WedelOptical GmbH upon OHB specifications with an accuracy of $<1.2 \mu \mathrm{rad}$ in each axis, calibrated traceable to national standards, and a resolution of $<1 \mu \mathrm{rad}$.

A special FAI monitoring system traced the angular stability of the FAI collimator system with respect to the FAI granite base. Long-term measurements of the granite monitoring against FAI collimator, FAI collimator against FAM, FAM against HDAC and HDAC against HSI proved an overall setup stability of better than $1 \mu$ rad over several hours.

FAI collimator WFE measurements were done using the FAM as a flat reference surface. A regular check of the FAI showed a stable WFE of $<60 \mathrm{~nm}$ rms over its 3 years of usage at OHB.

For the Knife-edge measurements (as well as for spectral characterization) a very homogeneous light field, both in real (focal plane-) space as well as in angular space, is desired. Figure 9 shows the illumination homogeneity in the focal plane of FAI measured with an Avalanche Photo Diode (APD). The two-dimensional spot in the X-Y plane was scanned along $\mathrm{X}$ for different $\mathrm{Y}$ positions. The measurement showed a perfect homogeneity of better $2 \% \mathrm{PV}$ due to the used fiber illumination.

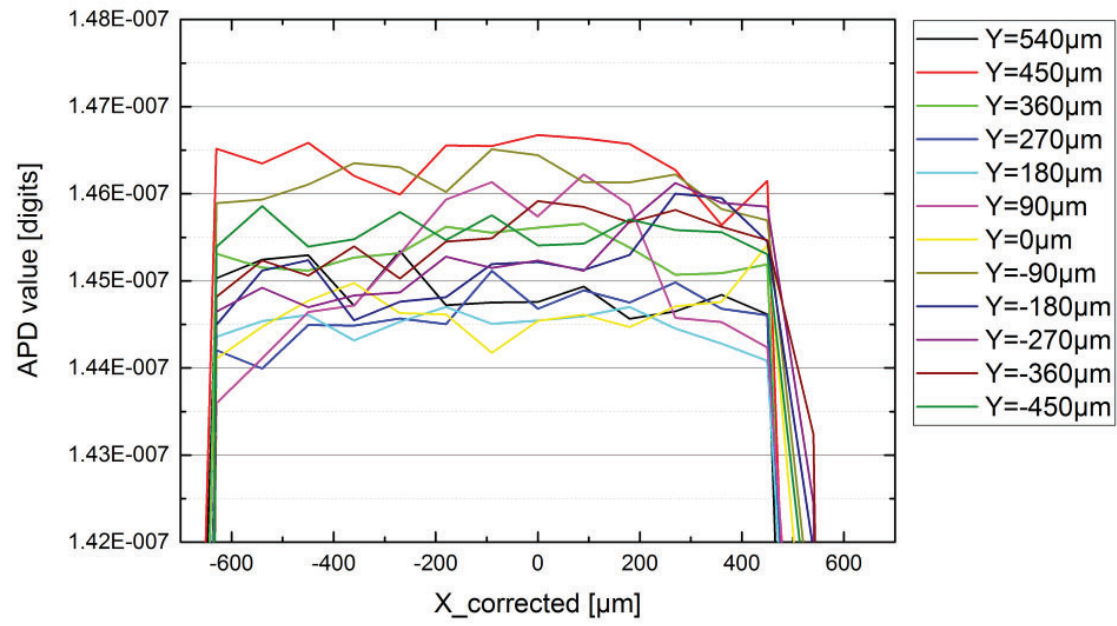

Figure 9. Illumination homogeneity in FAI focal plane, scanned with Avalance Photo Diode (APD).

To achieve high spectral calibration accuracy of HSI, the WiRAL had to deliver stable optical power during a scan range of at least $3 \mathrm{SSD}$ (ca. $5 \mathrm{~min}$ ). Figure 10 shows a stability measurement and analysis of WiRAL output power at 970nm over 3 hours. In Figure 10a, the evolution of the measured power $E$ is shown directly after switch on of the lamp. From the measured power values within a time interval of $5 \mathrm{~min}$ the trend and the normalized random noise (NRMSD) is calculated using the equations (1) to (3). 


$$
\begin{aligned}
\text { trend }[\%] & =\frac{\max (E)-\min (E)}{\operatorname{mean}(E)} * 100 \% \\
N R M S D[\%] & =\frac{\operatorname{RMSD}(E)}{\operatorname{mean}(E)} * 100 \% \\
\operatorname{RMSD}(E) & =\sqrt{\frac{\sum_{n=1}^{n}\left(\operatorname{mean}(E)-E_{n}\right)^{2}}{n}}
\end{aligned}
$$

Figure 10b shows the change in trend and NRMSD over time. After $0.6 \mathrm{~h}$ (36 $\mathrm{min})$ heat up time, the required stability of $0.5 \%$ trend and $0.2 \%$ NRMSD was achieved and dropped further to a stability of $<0.2 \%$ trend and $0.05 \%$ NRMSD for long term usage.

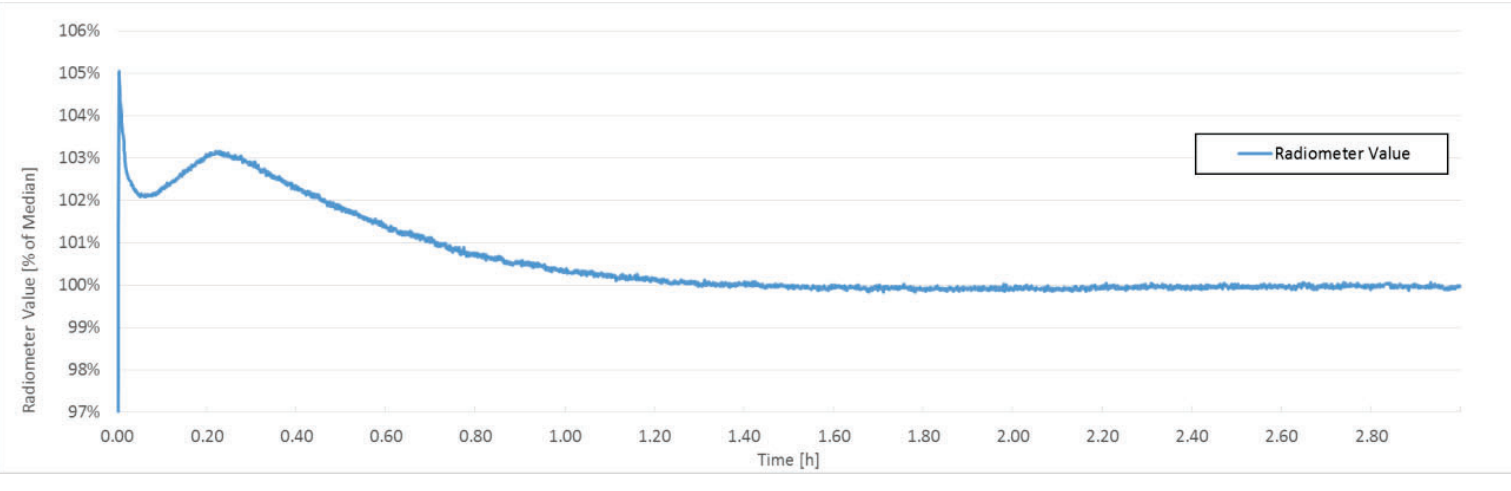

a)

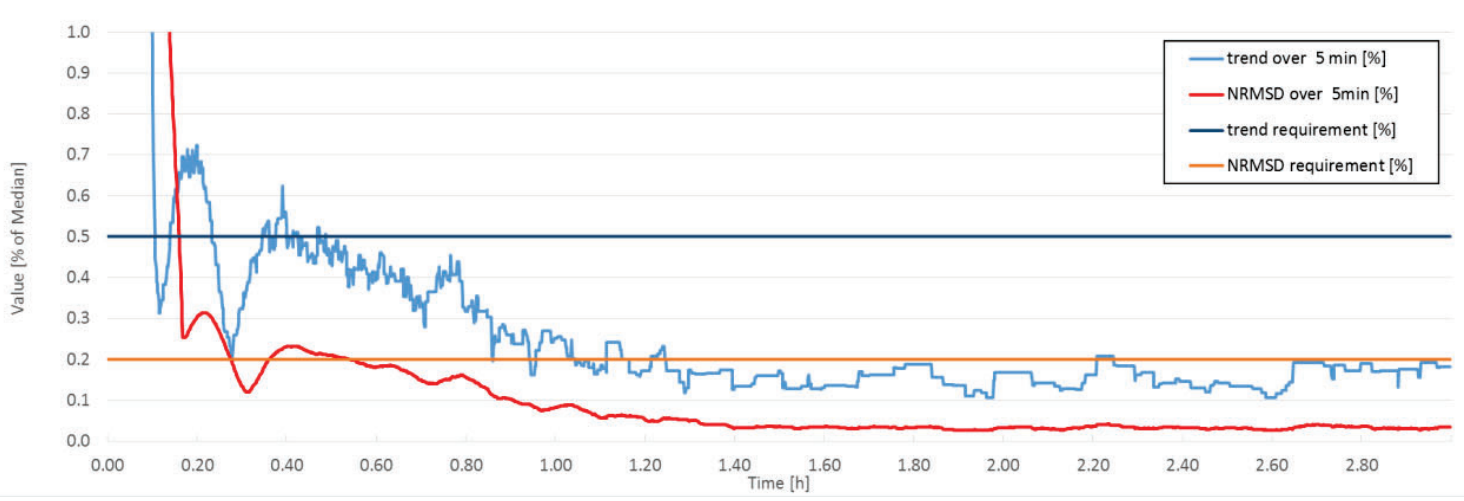

Figure 10. WiRAL output power stability at $970 \mathrm{~nm}$. a) relative optical power measured with a Si radiometer. b) Determined trend and NRMSD evolution over time.

\subsection{Straylight setup}

The setup for the straylight measurements was very similar to the setup for the spectral and geometrical measurements and is depicted in Figure 11. The FAI was used to illuminate the HSI together with WiRAL and the Stray Light Test Source (SLTS) from DLR-IMF as light sources. All GSE between FAI and instrument (HDAC, FAM) were removed to reduce possible stray light sources close to the EnMAP FOV. Additionally, a CAPE® tent with opaque fabric was set up around the setup to reduce parasitic light e.g. from small indicator LEDs of other devices in the room (see Figure 12).

As a very versatile OGSE, the FAI was designed right from the beginning to be also suitable for stray light measurements. The FAI scene generator was designed to allow special illumination scenes on the FSSA for stray light measurements, like $2 \times 2$ pixel illumination for the in-band stray light measurements or single pixel row illumination for spatial in-band stray light measurements. 


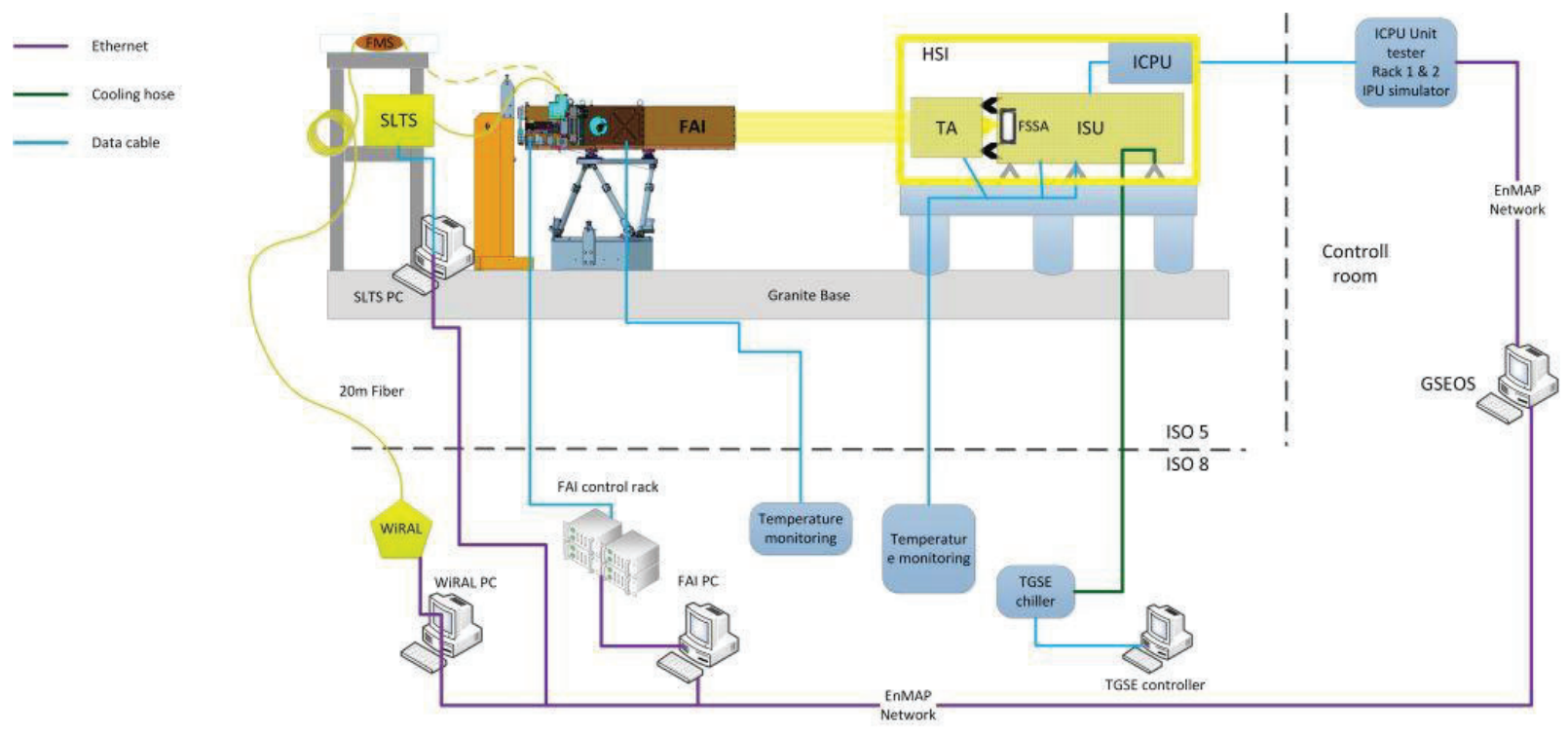

Figure 11. Stray light setup

The SLTS was designed to measure Point Spread Functions (PSF) at specific wavelengths with a very High Dynamic Range (HDR) [9]. These HDR PSFs can be used to correct the EnMAP Data for in-band stray light. Broadband illumination was possible as well, which was used for the out-of-field stray light characterization. Also the SLTS was controlled by the GSEOS software, which enabled automated measurements of HDR PSFs at numerous positions on the EnMAP detectors. On the VNIR detector, for example, HDR PSFs with a dynamic range of $10^{-9}$ were measured at 132 positions on the detector with a pure measurement time of about 16 hours in total.

For the spatial and spectral in-band ghost characterization, the WiRAL was used as a light source, because it delivered spectral narrow band illumination adjustable over the whole EnMAP range, as well as spectral broadband illumination optimized respectively for the two VNIR and SWIR spectrometer wavelength ranges.

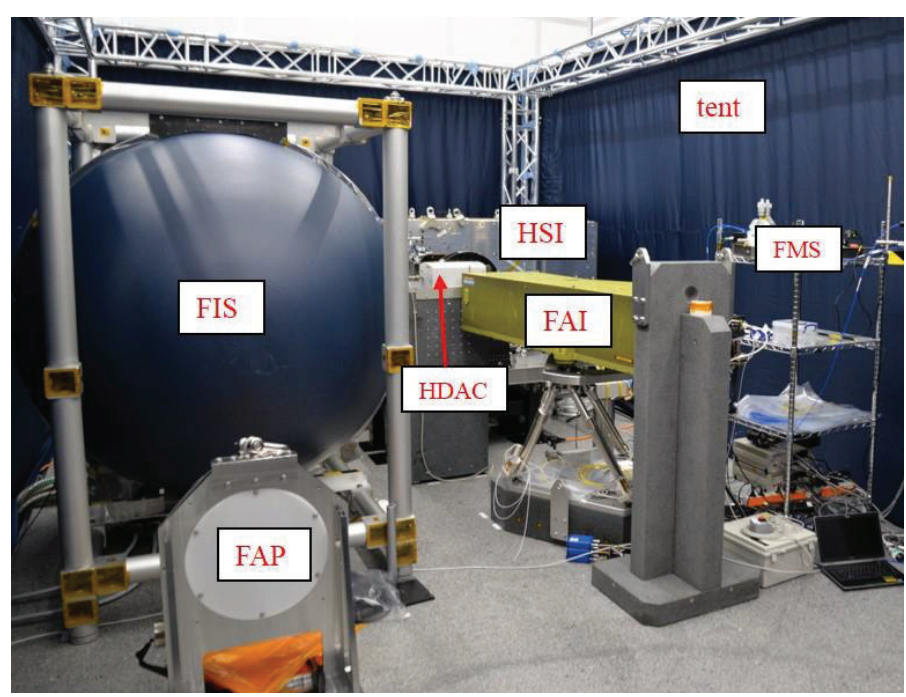

Figure 12. Picture of ISO5 cleanroom setup. 


\section{CONCLUSION}

We presented the OGSE setups and strategies used for alignment and characterization of the EnMAP hyperspectral instrument. The versatility and re-usage of OGSE for alignment and calibration allowed a deep understanding and characterization of the GSEs. In this way all measurements could be optimized, to finally guarantee the necessary high spectral and geometrical measurement accuracy from the instrument requirements. The Instrument data is actually still under evaluation, hence in this paper no statements on the instrument performance can be made.

\section{ACKNOWLEDGEMENTS}

The authors would like to thank A. Baumgartner, C. Rameloo and C. Köhler (DLR-IMF) for performing the spectral calibration activities of the WIRAL and the TS and assisting the radiometric and stray light measurements during CnC.

The work presented herein was performed on behalf of the German Space Agency DLR with funds of the German Federal Ministry of Economic Affairs and Technology under the grant No. 50 EP 0801.

\section{REFERENCES}

[1] L. Guanter, H. Kaufmann, K. Segl et al., "The EnMAP spaceborne imaging spectroscopy mission for earth observation," 7(7), 8830-8857 (2015).

[2] G. Huber, B. Sang, M. Erhard et al., "An all-silicon, high precision double-slit device for hyperspectral imager EnMAP." 11180, 1118011.

[3] M. Lettner, T. Lamour, L. Polz et al., "Versatile full aperture illumination OGSE Setup for alignment and endto-end calibration of the EnMAP hyperspectral image," 11180, 111806D (2019).

[4] J. Kolmeder, A. Kuisl, B. Sang et al., "Optical integration process for the earth-observing satellite mission ENMAP," 58 (2017).

[5] A. Godenir, J. Kolmeder, A. Kuisl et al., "Fine alignment of aerospace telescopes for the earth observation satellite mission ENMAP." 10562, 105624S.

[6] J. Brachmann, A. Baumgartner, and P. Gege, "The calibration home base for imaging spectrometers," 2(A82), 1-9 (2016).

[7] A. Baumgartner, and T. Schwarzmaier, [Traceable Radiometric Calibration of the German Imaging Spectrometer Satellite Mission EnMAP], (2017).

[8] A. Baumgartner, "Grating monochromator wavelength calibration using an echelle grating wavelength meter," 27(10), 13596-13610 (2019).

[9] C. Pope, and A. Baumgartner, "Light source for stray light characterisation of EnMAP spectrometers." 11151, 1115123. 